\title{
Materia oscura a partir del sector escalar en un modelo $U(1)_{X}$
}

\author{
Roberto Enrique Martínez-Martínez, Jorge Luis Nisperuza-Toledo*, Fredy Alexander Ochoa-Perez \\ Departamento de Física, Universidad Nacional de Colombia, Bogotá, Colombia
}

\begin{abstract}
Resumen
Considerando una extensión al modelo estándar de las partículas fundamentales del tipo $S U(3)_{C} \otimes S U(2)_{L} \otimes U$ $(1)_{Y} \otimes U(1)_{X}$, la cual contiene un escalar singlete como candidato a materia oscura, se estudian cotas a la masa de este escalar a partir de los datos de densidad reliquia para materia oscura provenientes del experimento Planck, los límites de la sección eficaz de dispersión DM-nucleón independiente de spin reportados por LUX, y las cotas de la fracción del ancho de decaimiento invisible del bosón de Higgs obtenidas en el colisionador LHC del CERN. Se obtienen regiones permitidas para la masa del escalar al considerar diferentes escenarios para el espacio de parámetros del modelo.
\end{abstract}

Palabras clave: modelo estándar, materia oscura, densidad reliquia.

Dark matter from scalar sector in a model

\begin{abstract}
By considering an extension of the standard model of fundamental particles like $S U(3)_{C} \otimes S U(2)_{L} \otimes U(1)_{Y} \otimes U(1)_{X}$, which contains a scalar singlet as a dark matter candidate, we study bounds to the mass of the scalar from the relic density data for dark matter from Planck experiment, the boundaries of the DM-nucleon spin independent scattering cross section reported by LUX, and bounds from the Higgs invisible decay width fraction obtained by the LHC collider at CERN. We obtain allowed regions for the mass of the scalar by consider different scenarios to model space parameters.
\end{abstract}

Key words: Standard model, dark matter, relic density.

\section{Introducción}

El enigma de la materia oscura (DM por sus siglas en inglés) en el Universo es uno de los principales temas de investigación de la física contemporánea. Actualmente existe un consenso bastante generalizado entre la comunidad científica el cual considera que el problema de DM concierne a la búsqueda de una partícula (o partículas) eléctricamente neutra y que interactúa débilmente, catalogada como WIMP por sus siglas del inglés para Weakly Interacting Massive Particle (Arneodo, 2013; Strigari, 2012). Esta búsqueda tradicionalmente se ha abordado a través de tres categorías que se complementan entre sí: detección directa, detección indirecta y búsqueda en aceleradores. La detección directa hace referencia a la dispersión elástica de partículas DM con núcleos en detectores que pueden medir la energía de retroceso del núcleo (nuclear recoil) del proceso. La detección indirecta busca cuantificar las partículas resultantes de la interacción de DM con la materia ordinaria a través de procesos de decaimiento o aniquilación de DM.

Por otra parte, diversas extensiones al modelo estándar de las partículas fundamentales (ME) han sido consideradas en la literatura, las cuales contienen partículas exóticas adicionales cuyas características podrían estar en concordancia con los datos experimentales de detección directa e indirecta. Entre esos modelos se tiene la extensión $S U(3)$ ${ }_{C} \otimes S U(2)_{L} \otimes U(1)_{Y} \otimes U(1)_{X}$, (Martinez, Nisperuza, Ochoa \& Rubio, 2014a), denotado $1_{X}$ en lo que sigue, la cual contiene un candidato escalar singlete $\sigma_{0}$ como posible candidato a DM. En la referencia (Martinez, Nisperuza, Ochoa \& Rubio, 2014b) se estudian algunas consecuencias fenomenológicas al considerar este escalar como partícula DM.

Este trabajo considera la simetría $1_{X}$ y un espacio de parámetros específicos de la misma con el fin de obtener cotas a la masa $M_{\sigma}$ del escalar $\sigma_{0}$. Estas regiones permitidas para $M_{\sigma}$ se hallan haciendo uso de los datos más recientes de densidad reliquia provenientes del experimento Planck (Planck Collaboration, Ade, et al., 2013), los límites de la sección eficaz de dispersión DM-nucleón independiente de spin reportados por LUX (LUX Collaboration, Akerib, et al., 2014), y las cotas de la fracción del ancho de decaimiento invisible del bosón de Higgs obtenidas en el colisionador LHC del CERN (ATLAS Collaboration, Aad, et al., 2014; CMS Collaboration, Chatrchyan, et al., 2014).

\footnotetext{
*Correspondencia:

Jorge Luis Nisperuza Toledo, jlnisperu@unal.edu.co

Recibido: 5 de junio de 2015

Aceptado: 21 de octubre de2015
} 


\section{Búsqueda de materia oscura a través de datos de detección directa e indirecta}

Una de las herramientas principales en la búsqueda de DM a través de detección indirecta, es el conocimiento del valor preciso de la densidad reliquia CDM provenientes del experimento Planck:

$$
\Omega_{\mathrm{CDM}} h^{2}=0.1199 \pm 0.0027
$$

Esta densidad reliquia está estrechamente relacionada con el promedio térmico de la sección eficaz de dispersión por la velocidad relativa $\langle\sigma v\rangle$ de DM con las partículas del ME. Específicamente, mediante el estudio de la evolución de una especie DM vía ecuación de Boltzmann, se tiene que:

$$
\Omega_{\psi} h^{2}=\frac{\left(1.07 \times 10^{9}\right) \chi_{f}}{\sqrt{g^{*}} m_{p l}(G e V)\langle\sigma v\rangle}
$$

siendo $m_{p l}$ la masa de Planck, $g^{*}$ los grados de libertad efectivos (los cuales son dependiente del modelo) y $x_{f}$ el parámetro $x=\frac{m}{T}$ evaluado en la temperatura de freeze-out, siendo $m$ la masa de la partícula DM y $T$ la temperatura. $\langle\sigma v\rangle$ contiene todas las interacciones de DM con las partículas del modelo y el cálculo de $\Omega_{\psi} h^{2}$ es llevado a cabo con el software micrOMEGAs (Belanger, Boudjema, Puknov \& Semenov, 2013).

Por otra parte, la idea principal de la detección directa de DM consiste en la posibilidad de detectar la energía de retroceso del núcleo originado de la interacción de DM con núcleos en procesos de dispersión. La sección eficaz para la amplitud de dispersión DM-nucleón en reposo se escribe (Seungwon, Ko, Park \& Wan, 2014; Del Nobile, et al., 2014)

$$
\sigma_{T}=\frac{4 M_{\sigma}^{2} M_{N}^{2}}{\pi\left(M_{\sigma}+M_{N}\right)^{2}}\left|A_{S I}\right|^{2}
$$

donde $M_{\sigma}$ es la masa de la partícula DM, $M_{N}$ la masa del nucleón blanco, mientras que $A_{S I}$ es la amplitud independiente de spin. En este trabajo se realiza el cálculo de esta cantidad a través del software micrOMEGAs.

En la literatura se encuentra el uso de datos de detección directa e indirecta, específicamente de $\Omega_{\psi} h^{2}$ y $\sigma_{T}$, para acotar el espacio de parámetros en modelos extendidos que consideran partículas escalares como candidatos DM, (ver por ejemplo Chang, Edezhath, Hutchinson \& Luty, 2014; Cogollo, et al., 2014; Nisperuza, 2015; Yaguna, 2011). También se tiene que los datos de detección directa han sido utilizados para la búsqueda de DM en extensiones mínimas del modelo estándar con un escalar singlete (Feng, Profumo \& Ubaldic, 2015) y en modelos supersimétricos (Ellis, Olive, Santoso \& Spanos, 2005).

Otra de las herramientas relevantes en detección directa es la fracción del ancho invisible de decaimiento del bosón de Higgs () del ME:

$$
\operatorname{Br}\left(h_{0} \rightarrow \text { inv. }\right)=\frac{\Gamma\left(h_{0} \rightarrow \text { inv. }\right)}{\Gamma\left(h_{0} \rightarrow \text { todas. }\right)},
$$

la cual impone límites al decaimiento de DM a través del Higgs del ME. Los datos más recientes son reportados por las colaboraciones ATLAS (ATLAS Collaboration, Aad, et al., 2014) y CMS (CMS Collaboration, Chatrchyan, et al., 2014), indicando $\mathrm{Br}\left(h_{0} \rightarrow\right.$ inv. $)<0.58$ al $95 \%$ C.L. y $\mathrm{Br}$ $\left(h_{0} \rightarrow\right.$ inv. $)<0.75$ al $95 \%$ C.L., respectivamente.

Las referencias (Sanabria, 2014; Shafi, Hanif \& Salih, 2015) muestran un panorama adicional sobre el estado actual de la búsqueda de DM a través de datos de detección directa con LCH.

\section{Modelo $S U(3)_{C} \otimes S U(2)_{L} \otimes U(1)_{Y} \otimes U(1)_{X}$}

\section{Contenido de partículas.}

A continuación se describe el conjunto completo de partículas del modelo, el cual incluye todas las partículas ME y también nuevas partículas exóticas.

$$
\begin{gathered}
\tilde{\mathrm{q}}_{L}^{n}=i \sigma_{2} q_{L}^{n}=\left(\begin{array}{c}
U^{n} \\
D^{n}
\end{array}\right)_{L} \sim\left(2, \frac{1}{3}, 0\right) ; J_{L}^{n} \sim\left(1,-\frac{2}{3}, 0\right) \\
J_{L}^{n} \sim\left(1,-\frac{2}{3},-\frac{1}{3}\right) \\
\mathrm{q}_{L}^{3}=\left(\begin{array}{c}
U^{3} \\
D^{3}
\end{array}\right)_{L} \sim\left(2, \frac{1}{3}, \frac{1}{3}\right) ; T_{L}^{n} \sim\left(1, \frac{4}{3}, \frac{1}{3}\right) \\
T_{R}^{n} \sim\left(1, \frac{4}{3}, \frac{2}{3}\right) \\
U_{R}^{i} \sim\left(1, \frac{4}{3}, \frac{2}{3}\right) ; D_{R}^{i} \sim\left(1,-\frac{2}{3},-\frac{1}{3}\right) ; \\
l_{R}^{i} \sim\left(2,-1,-\frac{1}{3}\right) ;\left(v_{R}^{i}\right)^{c} \sim\left(1,0,-\frac{1}{3}\right) ; \\
e_{R}^{i} \sim(1,-2,-1) ; N_{R}^{i} \sim(1,0,0) ;
\end{gathered}
$$

donde $i=1,2,3$ y $n=1$, 2 son índices de familia mientras que $\left(S U(2)_{L}, U(1)_{Y}, U(1)_{X}\right)$ denota las reglas de transformación para el modelo.

El contenido de fermiones nuevos consta de los quarks singletes $T$ y $J^{n}$, los cuales son de tipo up y down, respectivamente. Adicionalmente, se tienen los neutrinos $\left(v_{R}^{i}\right)^{c} \mathrm{y}$ $N_{R}^{i}$, los cuales se incluyen para el estudio de la generación de masas de neutrinos.

Para los campos escalares se construyen dobletes y singletes de $S U(2)_{L}$,

$$
\begin{aligned}
& \chi_{0}=\frac{1}{\sqrt{2}}\left(v_{\chi}+\xi_{\chi}+i \varsigma_{\chi}\right) \sim\left(1,0,-\frac{1}{3}\right) ;\left\langle\chi_{0}\right\rangle_{0}=v_{\chi} \\
& \phi_{1}=\left(\begin{array}{c}
\phi_{1}^{+} \\
\frac{1}{\sqrt{2}}\left(v_{1}+\xi_{1}+i \phi_{1}^{0}\right)
\end{array}\right) \sim\left(2,1,-\frac{2}{3}\right) ;\left\langle\phi_{1}\right\rangle_{0}=v_{1} \\
& \phi_{2}=\left(\begin{array}{c}
\phi_{2}^{+} \\
\frac{1}{\sqrt{2}}\left(v_{2}+\xi_{2}+i \phi_{2}^{0}\right)
\end{array}\right) \sim\left(2,1,-\frac{1}{3}\right) ;\left\langle\phi_{2}\right\rangle_{0}=v_{2} \\
& \sigma_{0}=\frac{1}{\sqrt{2}}\left(v_{\sigma}+\xi_{\sigma}+i \varsigma_{\sigma}\right) \sim\left(1,0,-\frac{1}{3}\right) ;\left\langle\sigma_{0}\right\rangle_{0}=v_{\sigma}
\end{aligned}
$$


De los anteriores campos, $\phi_{2}$ es un nuevo escalar doblete, el cual es necesario para obtener fermiones cargados masivos; $\chi_{0}$ y $\sigma_{0}$ son campos adicionales singletes, donde $\sigma_{0}$ es el responsable del rompimiento de la simetría $1_{X}$ para lo cual es necesario imponer la jerarquía $v_{\gamma} \gg v$. El nuevo campo $\sigma_{0}$ no es esencial para el rompimiento espontáneo de la simetría, por lo que se escoge $v_{\sigma}=0$ para convertirlo en el candidato a DM de este trabajo. Finalmente, los campos de gauge del modelo son los siguientes,

$$
\begin{gathered}
W_{\mu}=\left(\begin{array}{cc}
W_{\mu}^{3} & \sqrt{ } 2 W_{\mu}^{+} \\
\sqrt{ } 2 W_{\mu}^{-} & -W_{\mu}^{3}
\end{array}\right) \sim(3,0,0) \\
B_{\mu}=\sim(0,1,0) \\
Z_{\mu}^{\prime}=\sim(0,0,1),
\end{gathered}
$$

siendo $Z_{\mu}^{\prime}$ un nuevo bosón de gauge asociado a la simetría $U(1)_{X}$.

\section{Sector Escalar.}

Se muestra aquí una versión adecuada del potencial escalar del modelo, indicado en la referencia Martinez, et al., (2014a). Este potencial tiene en cuenta al escalar $\sigma_{0}$ como candidato a DM y es construido introduciendo la simetría global $\sigma_{0} \rightarrow \mathrm{e}^{i \theta} \sigma_{0}$ en la expresión más general del potencial para $1_{X}$, renormalizable e invariante $S U(3)_{C} \otimes S U(2)_{L} \otimes U(1)_{Y}$ $\otimes U(1)_{X}$,

$$
\begin{gathered}
V_{H}=\mu_{1}^{2}\left|\phi_{1}\right|^{2}+\mu_{2}^{2}\left|\phi_{2}\right|^{2}+\mu_{3}^{2}\left|\chi_{0}\right|^{2}+\mu_{4}^{2}\left|\sigma_{0}\right|^{2}+f_{2}\left(\phi_{2}^{\dagger} \phi_{1} \chi_{0}+h . c\right) \\
+\lambda_{1}\left|\phi_{1}\right|^{4}+\lambda_{2}\left|\phi_{2}\right|^{4}+\lambda_{3}\left|\chi_{0}\right|^{4}+\lambda_{4}\left|\sigma_{0}\right|^{4} \\
+\left|\phi_{1}\right|^{2}\left(\lambda_{6}\left|\chi_{0}\right|^{2}+\lambda_{6}^{\prime}\left|\sigma_{0}\right|^{2}\right)+\left|\phi_{2}\right|^{2}\left(\lambda_{7}\left|\chi_{0}\right|^{2}+\lambda_{7}^{\prime}\left|\sigma_{0}\right|^{2}\right) \\
+\lambda_{5}\left|\phi_{1}\right|^{2}\left|\phi_{2}\right|^{2}+\lambda_{5}^{\prime}\left|\phi_{1}^{\dagger} \phi_{2}\right|^{2}+\lambda_{8}\left|\chi_{0}\right|^{2}\left|\sigma_{0}\right|^{2}
\end{gathered}
$$

Es importante notar que el anterior potencial no contiene términos impares de $\sigma_{0}$ lo cual evita el decaimiento directo de la materia oscura en otras partículas escalares.

\section{Acoples de DM con los bosones de gauge neutros.}

El sector cinético del lagrangiano de Higgs se escribe

$\mathcal{L}_{K}=i \sum_{i=1,2}\left(D_{\mu}^{D} \phi_{i}\right)^{\dagger}\left(D^{\mu D} \phi_{i}\right)+\left(D_{\mu}^{S} \sigma_{0}\right)^{\dagger}\left(D^{\mu S} \sigma_{0}\right)+\left(D_{\mu}^{S} \chi_{0}\right)^{\dagger}\left(D^{\mu S} \chi_{0}\right)$

donde $D_{\mu}^{D}=\partial_{\mu}-i g W_{\mu}^{\alpha} T_{\alpha}-i g^{\prime} \frac{Y}{2} B_{\mu}-i g_{X} X Z_{\mu}^{\prime}$ y $D_{\mu}^{s}=\partial_{\mu}-i g^{\prime} \frac{Y}{2}$ $B_{\mu}-i g_{X} X Z_{\mu}^{\prime}$ denotan las derivadas covariantes para los escalares dobletes y singletes, siendo $g, g$, y $g_{X}$ las constantes de acople asociadas a $S U(2)_{L}, U(1)_{Y}$ y $U(1)_{X}$, respectivamente; los $W_{\mu}^{\alpha}$ son los 3 bosones de gauge asociados a $S U(2)_{L}$; $2 T_{\alpha}$ son las matrices de Pauli; $Y$ y $X$ corresponden a la hipercarga y a la carga bajo el grupo $U(1)_{X}$, y los campos $B_{\mu}$ y $Z_{\mu}^{\prime}$ son los bosones de gauge asociados a las simetrías $U(1)_{Y}$ y $U(1)_{X}$, respectivamente.

Con el rompimiento espontáneo de la simetría y luego de efectuar las rotaciones surge el autoestado neutro no masivo correspondiente al fotón $A_{\mu}$, los autoestados cargados $\mathrm{W}^{ \pm}=$ $\left(\mathrm{W}_{\mu}{ }^{1} \pm \mathrm{W}_{\mu}{ }^{2}\right) / \sqrt{ } 2$ con masa $\mathrm{M}_{W}=g v / 2$. También se tienen los autoestados $Z$ del ME y el nuevo bosón $Z$ ' cuyas masas, a orden dominante $\left(\left(v / v_{\chi}\right)^{2}<<1\right)$, son $\mathrm{M}_{Z} \approx \frac{g v}{2 C_{W}}$ y $\mathrm{M}_{Z^{\prime}} \approx \frac{g_{X} v_{\chi}}{2 C_{W}}$, siendo $\tan \theta_{W}=\frac{g^{\prime}}{g}$ el ángulo de Weinberg.

\section{Lagrangianos de Yukawa y de Dirac.}

El lagrangiano de Dirac, el cual describe las interacciones entre los fermiones y los bosones de gauge, se escribe

$$
\mathcal{L}_{D}=i \sum_{f, i} \overline{f_{L}} \gamma^{\mu} D_{\mu} f_{L}^{i}+\overline{f_{R}^{l}} \gamma^{\mu} D_{\mu} f_{R}^{i},
$$

donde las $\overline{f_{L, R}^{l}}$ contienen fermiones del modelo $1_{X}$. En la referencia Martinez, et al., (2014a) este lagrangiano es expandido para el sector débil neutro y es escrito en términos de acoples vectoriales y axiales. En este trabajo se obtienen todos los acoples directamente a través del paquete computacional LanHEP (Semenov, 2010).

Por otra parte, el potencial de Yukawa más general que se puede construir para el modelo $1_{X}$ se describe también en la referencia Martinez, et al., (2014a). Para la búsqueda de DM, y con el fin de simplificar el análisis, se asumen matrices de Yukawa diagonales. Con esto, el lagrangiano de Yukawa se escribe como

$$
\begin{gathered}
-\mathcal{L}_{Y}=\overline{q_{L}^{l}}\left(\tilde{\phi}_{1} h_{1}^{U}+\tilde{\phi}_{2} h_{2}^{U}\right)_{i j} U_{\mathrm{R}}^{j}+\overline{q_{L}^{l}}\left(\phi_{1} h_{1}^{D}+\phi_{2} h_{2}^{D}\right)_{i j} D_{\mathrm{R}}^{j} \\
+\overline{q_{L}^{l}}\left(\phi_{1} h_{1}^{J}+\phi_{2} h_{2}^{J}\right)_{i m} J_{\mathrm{R}}^{m} \\
+\overline{J_{L}^{n}}\left(\chi_{0} h_{\chi}^{D}\right)_{n j} D_{R}{ }^{j} \overline{J_{L}^{n}}\left(\sigma_{0} h_{\sigma}^{J}+\chi_{0} h_{\chi}^{J}\right)_{n m} J_{R}^{m}+h \cdot c \\
+\overline{l_{L}^{l}}\left(\tilde{\phi}_{1} h_{1}^{v}\right)_{i j} v_{R}^{j}++\overline{l_{L}^{l}}\left(\tilde{\phi}_{2} h_{1}^{J}\right)_{i j} v_{R}^{j}+\overline{\left(v_{R}^{l}\right)^{c}}\left(\chi_{0}^{*} h_{\chi}\right)_{i j} N_{R}^{i} \\
+\frac{1}{2} M_{N} \overline{\left(N_{R}^{l}\right)^{c}} N_{R}^{j}+\overline{l_{L}^{l}}\left(\phi_{1} h_{1}^{e}\right)_{i j} e_{R}^{j}+h . c,
\end{gathered}
$$

donde $\tilde{\phi}_{1,2}=i \sigma_{2} \phi_{1,2}^{*}$.

\section{Restricciones provenientes de $\Omega h^{2}, \operatorname{Br}\left(h_{0} \rightarrow i n v.\right)$ y detección directa}

\section{Implementación de cálculo computacional.}

El código numérico MicrOMEGAs permite el cómputo de $\Omega \mathrm{h}^{2}, T_{f} \mathrm{y}\langle\sigma v\rangle$, ec. (2), previo ingreso del modelo $1_{X}$. Para esto último se hizo uso del paquete LanHEP, el cual genera todas las reglas del Feynman para las interacciones entre las partículas del modelo. Internamente MicrOMEGAs asume que la abundancia de la especie DM satisface la condición $Y_{e q} \approx Y(T)$ para altas temperaturas hasta la temperatura de freeze-out, y $Y_{\text {eq }}=0$ para temperaturas por debajo de la temperatura de freeze-out.

Se toma $M_{H 0}=500 \mathrm{GeV}$ puesto que no se ha reportado a la fecha la existencia de un bosón de Higgs neutro adicional, y, $M_{h 0}=125.5 \mathrm{GeV}$ según lo reportado por ATLAS y CMS. Así, se tiene que para la dispersión indirecta en partículas escalares en procesos de la forma $\sigma_{0} \sigma_{0}^{*} \rightarrow S(2 S)$, acorde a las restricciones indicadas anteriormente, los únicos parámetros que quedan libres son las constantes de acople $\lambda_{6}{ }_{6} \mathrm{y} \lambda_{7}{ }_{7}$. Para el campo de gauge $Z$ ' se toma una masa del orden de 3 TeV y un ángulo de mezcla $Z-Z$ ', $0 \leq S_{\theta} \leq 0.4 \times 10^{-3}$. El ángulo de mezcla $\alpha$ entre $h_{0}-H_{0}$ está dado por (Martinez, et al., 2014b.) 


$$
\tan 2 \alpha=\tan 2 \beta\left(1-\frac{\mathrm{M}_{h 0}^{2}}{\mathrm{M}_{H 0}^{2}}\right)
$$

Con todas las consideraciones anteriores, el espacio de parámetros del modelo queda finalmente compuesto de solo cuatro variables $\left(M_{\sigma}, \mathrm{T}_{\beta}, \lambda_{6}^{\prime}, \lambda_{7}^{\prime}\right)$.

\section{Límites para $\mathrm{M \sigma}$.}

Con el fin de explorar el efecto de las restricciones provenientes de los datos de detección directa e indirecta de DM, se toma el caso más general en el cual $0 \leq \mathrm{T}_{\beta} \leq 10$ y $0 \leq \lambda_{6,7}^{\prime} \leq 4 \pi$, ambos aleatorios. La región escogida para $\lambda_{6,7}^{\prime}$ corresponde al límite de unitariedad para los acoples escalares. La figura 1 muestra la densidad reliquia como función de la masa del candidato escalar $\mathrm{DM}$ con $\mathrm{Br}\left(h_{0} \rightarrow i n v\right.$ ) $\leq 0.3$ (Figura 1a) y $\mathrm{Br}\left(h_{0} \rightarrow i n v\right.$. $) \leq 0.1$ (Figura $1 \mathrm{~b}$ ), ambos en el rango de los límites experimentales indicados en la sección 1. La línea horizontal es el límite experimental para la densidad reliquia proveniente de Planck. En ambas figuras, las marcas D, E, A y $\mathbf{H}$ indican threshold cinemáticos. F, G y $\mathbf{B}$ son resonancias debidas a la producción de partículas intermediarias. Más específicamente, $\mathbf{A}$ denota el threshold electrodébil $\mathrm{M}_{\sigma} \approx 91 \mathrm{GeV} ; \mathrm{M}_{\sigma} \approx 1.3 \mathrm{GeV}$ (D) es debido a la dispersión de $\sigma_{0}$ en los quarks charm, mientras que $M_{\sigma}$ $\approx 4 \mathrm{GeV}(\mathbf{E})$ es debida a la producción de quarks bottom. La resonancia en $M_{\sigma}=\frac{\mathrm{M}_{h 0}}{2}=63 \mathrm{GeV}(\mathbf{F})$ corresponde a los procesos $\sigma_{0} \sigma_{0}^{*} \rightarrow h_{0} \rightarrow M E, M E$ a través del bosón de Higgs tipo $\mathrm{ME}$; el pico de resonancia debido al polo $(2 M \sigma)^{2}-$ $\left(M_{H 0}\right)^{2}=0$, se indica en $\mathbf{G}$ mientras que en $M_{\sigma}=1500 \mathrm{GeV}$ (denotado como B) se tiene la resonancia $\left(2 M_{\sigma}\right)^{2}-M_{Z^{\prime}}^{2}=0$, en la cual claramente la probabilidad de aniquilación crece significativamente debido a procesos de la forma $\sigma_{0} \sigma_{0}^{*} \rightarrow \mathrm{Z}^{\prime} \rightarrow M E, M E$. Es posible apreciar que existen puntos solución para la densidad reliquia en el rango $1.5 \mathrm{GeV} \leq$ $M_{\sigma} \leq 65 \mathrm{GeV}$. Aunque la forma funcional de la distribución de puntos es la misma para ambas figuras, se observa que la restricción $\mathrm{Br}\left(h_{0} \rightarrow\right.$ inv. $) \leq 0.3$ (Figura 1a.) conlleva a una densidad de puntos mayor en la región permitida, en comparación a (Figura 1b.).

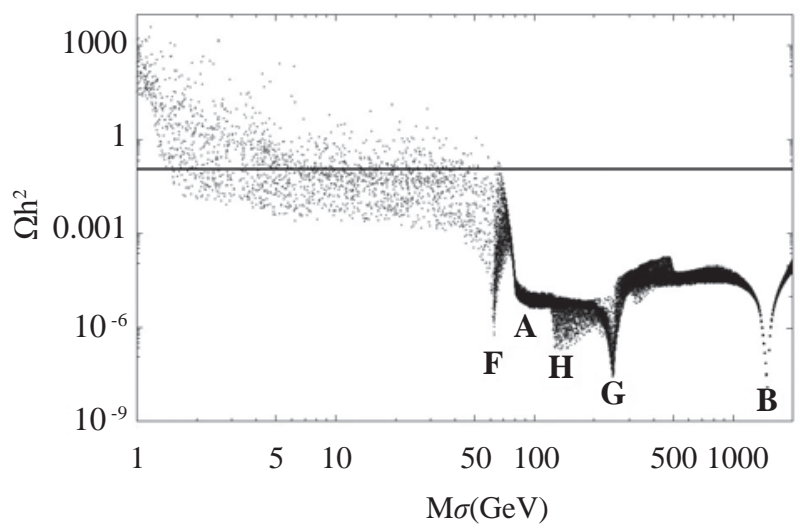

\section{Restricciones Provenientes de Datos de Detección Directa.}

Se exploran ahora límites provenientes de los datos más recientes de detección directa de LUX. Se calcula con micrOMEGAs la sección eficaz de dispersión WIMPnucleón, imponiendo la restricción $\mathrm{Br}\left(h_{0} \rightarrow i n v\right.$. $) \leq 0.3$. Todos los puntos satisfacen $\Omega h^{2} \sim 0.11$.

La figura 2 muestra los resultados para $0 \leq \lambda_{6,7}^{\prime} \leq 4 \pi, 0 \leq \mathrm{T}_{\beta} \leq$ 10 y $0 \leq \mathrm{S}_{\theta} \leq 0.4 \times 10^{-3}$, todos aleatorios. La línea punteada es el límite actual de LUX. Se observa que es clara la tendencia a tener puntos no excluidos por LUX en $M_{\sigma} \approx 63 \mathrm{GeV}$, es decir, a medida que nos acercamos a la resonancia del Higgs del ME.

\section{Conclusiones}

Este trabajo aborda el problema de la búsqueda de DM a partir de un modelo extendido $1_{X}$ con un candidato escalar singlete $\sigma_{0}$. Se estudió el efecto de los datos de detección directa e indirecta de DM sobre el conjunto de parámetros del modelo, considerando escenarios específicos, de lo cual se obtuvo lo siguiente:

- Mediante las restricciones provenientes de Planck y los límites de la fracción del ancho de decaimiento del bosón de Higgs de ME, se obtiene una región permitida para $M_{\sigma}$ en el rango $1.5 \mathrm{GeV} \leq M_{\sigma} \leq 65 \mathrm{GeV}$.

- Al considerar el límite experimental para la sección eficaz de dispersión DM-nucleón, se encuentra que el modelo posee puntos no excluidos en la región $10 \mathrm{GeV} \leq M_{\sigma} \leq 65$ $\mathrm{GeV}$, por lo que esta restricción complementa los límites encontrados de datos de detección indirecta y del ancho invisible de decaimiento del bosón de Higgs del ME.

Por lo tanto, los datos de detección directa e indirecta en la búsqueda de DM tienen un

impacto significativo sobre el espacio de parámetros del modelo $1_{X}$, en particular, se obtiene una región permitida para la masa de DM de $10 \mathrm{GeV} \leq M_{\sigma} \leq 65 \mathrm{GeV}$.

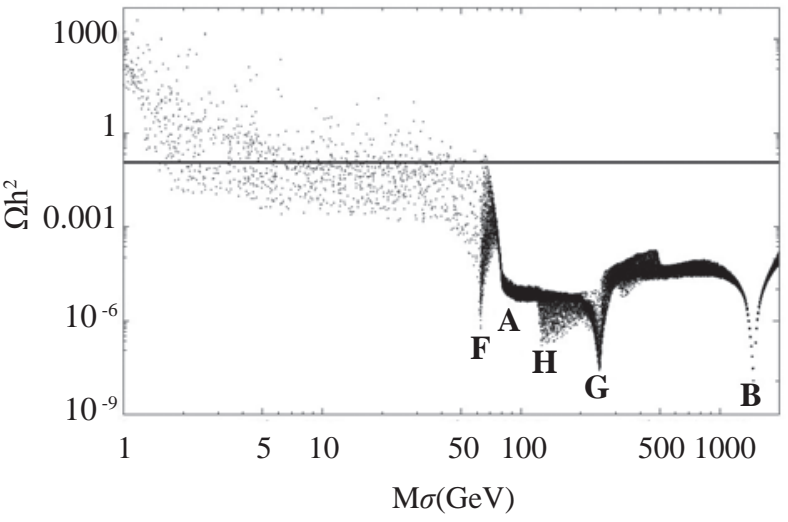

Figura 1. Densidad reliquia como función de $M_{\sigma}$ considerando: (a) $B r\left(h_{0} \rightarrow\right.$ inv. $) \leq 0.3$ y (b) $B r\left(h_{0} \rightarrow i n v\right.$. $) \leq 0.1$, donde $0 \leq \lambda_{6,7}^{\prime} \leq 4 \pi$, $0 \leq \mathrm{T}_{\beta}$ $\leq$ 10. Las marcas D, E, A, y $\mathbf{H}$ indican threshold cinemáticos. F, G y B son resonancias debidas a la producción de partículas intermediarias. 


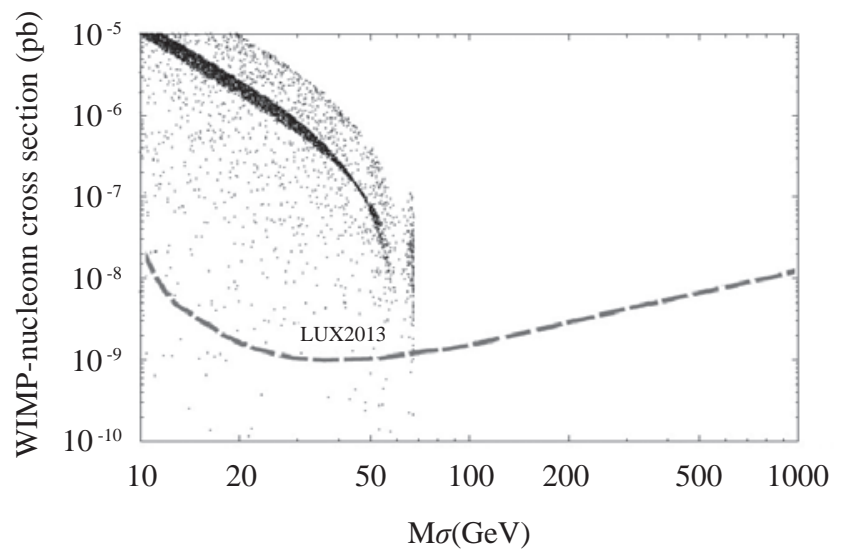

Figura 2. WIMP-nucleon SI vs. $M_{\sigma} .0 \leq \lambda_{6,7}^{\prime} \leq 4 \pi, 0 \leq \mathrm{T}_{\beta} \leq 10$ y 0 $\leq \mathrm{S}_{\theta} \leq 0.4 \times 10^{-3} \cdot \mathrm{Br}\left(h_{0} \rightarrow\right.$ inv. $) \leq 0.3$. La línea punteada es el límite actual de LUX.

\section{Agradecimientos}

Éste trabajo está financiado por el programa El Patrimonio Autónomo Fondo Nacional de Financiamiento para la Ciencia, la Tecnología y la Innovación Fransisco José de Caldas de COLCIENCIAS.

\section{Conflicto de intereses}

Los autores manifiestan que no tienen conflicto de intereses.

\section{Bibliografía}

Arneodo F. 2013. Dark Matter Searches. arXiv:1301.0441v1 [astro-ph.IM].

ATLAS Collaboration, Aad, et al. 2014. Search for Invisible Decays of a Higgs Boson Produced in Association with a Z Boson in ATLAS. Phys. Rev. Lett. 112: 201802.

Belanger G., Boudjema F., Pukhov A., Semenov A. 2013. micrOMEGAs3.1: a program for calculating dark matter observables. arXiv: 1305.0237 [hep-ph].

Chang S., Edezhath R., Hutchinson J., Luty M. 2014. Effective WIMPs. Phys. Rev. D 89: 015011

CMS Collaboration, Chatrchyan, et al. 2014. Search for invisible decays of Higgs bosons in the vector boson fusion and associated ZH production modes. The European Physical Journal C. 74: s10052-014-2980-6.
Cogollo D., Gonzalez-Morales A., Queiroz F., Rebello P. 2014. Excluding the light dark matter window of a 331 model using LHC and direct dark matter detection data. arXiv: 1402.3271v2 [hep-ph].

Del Nobile E., Gelmini G., Gondolo P., Huh Ji-Haeng. 2014. Update on the Halo-Independent Comparison of Direct Dark Matter Detection Data. arXiv: 1405.5582v1 [hep-ph].

Ellis J., Olive K., Santoso Y., Spanos V. 2005. Update on the direct detection of supersymmetric dark matter. Phys. Rev. D71: 095007.

Feng L., Profumo, S., Ubaldic L. 2015. Closing in on singlet scalar dark matter: LUX, invisible Higgs decays and gamma-ray lines. JHEP 03 (2015) 045.

LUX Collaboration, Akerib, et al. 2014. First Results from the LUX Dark Matter Experiment at the Sanford Underground Research Facility. Phys. Rev. Lett. 112: 091303.

Martinez R., Nisperuza J., Ochoa F., Rubio J. 2014a. Some phenomenological aspects of a new U(1)' model. Phys. Rev. D 89: 056008.

Martinez R., Nisperuza J., Ochoa F., Rubio J. 2014b. Scalar dark matter with CERN-LEP data and Z' search at the LHC in an U(1)' model. Phys. Rev. D 90: 095004.

Nisperuza J. 2015. Candidatos a materia oscura en modelos 331. Tesis Doctoral. Universidad Nacional de Colombia.

Planck Collaboration, Ade, et al. 2013. Planck 2013 results. XVI. Cosmological parameters. arXiv:1303.5076 [astro-ph.CO].

Sanabria J. 2014. Búsquedas de Materia Oscura Supersimétrica en el LHC. Rev. Acad. Colomb. Cienc. 38 (2014) 34-55.

Shafi Q., Hanif S., Salih C. 2015. Neutralino Dark Matter and Other LHC Predictions from Quasi Yukawa Unification. Nucl. Phys. B900 (2015) 400-411.

Semenov A. 2010. LanHEP-a package for automatic generation of Feynman rules from the Lagrangian. Updated version 3.1. arXiv: 1005.1909 [hep-ph].

Seungwon B., Ko P., Park Wan-II. 2014. Invisible Higgs decay width versus dark matter direct detection cross section in Higgs portal dark matter models. Phys. Rev. D 90: 055014.

Strigari L. 2012. Galactic Searches for Dark Matter. Physics Reports. doi 10.1016/j.physrep.2013.05.004

Yaguna C. 2011. The Singlet Scalar as FIMP Dark Matter. JHEP 1108 (2011) 060. 